\title{
Size-Dependent Pressure-Response of the
}

\section{Photoluminescence of $\mathrm{CsPbBr}_{3}$ Nanocrystals}

\section{- Supporting Information}

\section{J. Curtis Beimborn II, Luke R. Walther, Kenneth D. Wilson, J. Mathias Weber*}

JILA and the Department of Chemistry, University of Colorado, Boulder, CO 80309-0440, USA.

\section{Table of Contents}

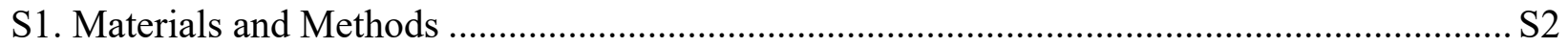

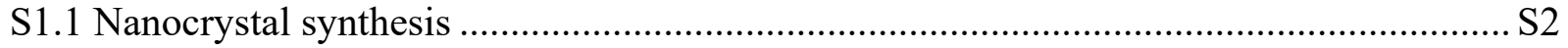

S1.2 Photoluminescence spectroscopy ………………………………………………....... S3

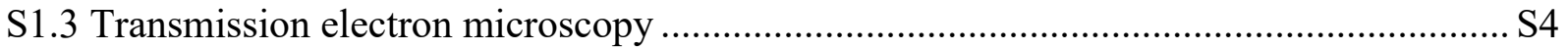

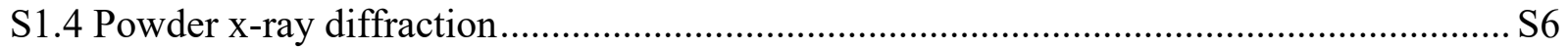

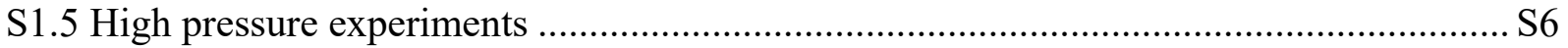

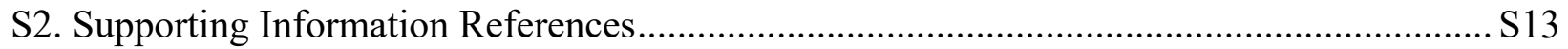

\section{Table of Figures}

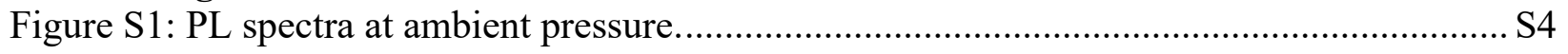

Figure S2: Size distributions and characteristic sample TEM images...................................... S5

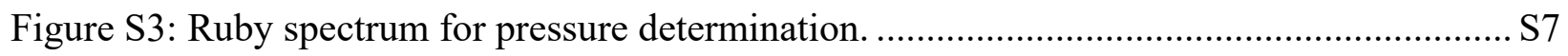

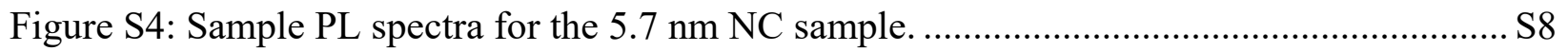

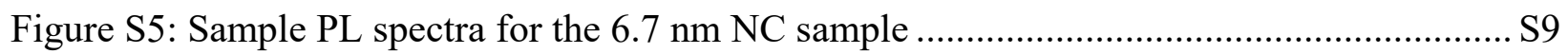

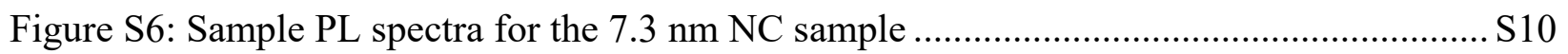

Figure S7: Sample PL spectra for the $7.9 \mathrm{~nm}$ NC sample ................................................... S1

Figure S8: Sample PL spectra for the $10.9 \mathrm{~nm}$ NC sample ................................................... S12 


\section{S1. Materials and Methods}

\section{S1.1 Nanocrystal synthesis}

We synthesized $\mathrm{CsPbr}_{3} \mathrm{NCs}$ following previously established hot injection methods. ${ }^{1-3}$ We tuned the size of the $\mathrm{NCs}$ by controlling the $\mathrm{Br}^{-}: \mathrm{Pb}^{2+}$ ratio, using oleylammonium bromide (OLA-Br). $\mathrm{CsPbBr}_{3} \mathrm{NCs}$ have $\mathrm{Br}^{-}$rich surfaces, so as the $\mathrm{Br}^{-}: \mathrm{Pb}^{2+}$ ratio increases, smaller $\mathrm{NCs}$ with larger surface to volume ratios are thermodynamically favored. ${ }^{1,3}$ This removes the difficulty of trying to control the NC size through kinetics. The OLA-Br was synthesized using $10 \mathrm{~mL}$ of purified oleylamine (OLA) ${ }^{4}$ and $1 \mathrm{~mL}$ of $\mathrm{HBr}$ (Sigma-Aldrich, reagent grade 48\%).

In a typical synthesis, $75 \mathrm{mg}$ of $\mathrm{PbBr}_{2}$ (Aldrich, $\geq 98 \%$ ), $5 \mathrm{~mL}$ of 1-octadecene (ODE) (Aldrich, technical grade 90\%), $0.5 \mathrm{~mL}$ of oleic acid (OA) (Aldrich, technical grade 90\%), and a total of $0.5 \mathrm{~mL}$ of OLA and OLA-Br were added to a $25 \mathrm{~mL}$ three neck round bottom flask. We changed the $\mathrm{Br}^{-}: \mathrm{Pb}^{2+}$ ratio by adjusting the OLA-Br:OLA ration while keeping the final volume consistent across all syntheses.

In a separate $25 \mathrm{~mL}$ round bottom flask, we added $162.5 \mathrm{mg}$ of $\mathrm{Cs}_{2} \mathrm{CO}_{3}$ (Aldrich, $99 \%$ ), 9 $\mathrm{mL}$ of ODE and $1 \mathrm{~mL}$ of OA to make a Cs-oleate.

Both flasks were stirred and heated to $120^{\circ} \mathrm{C}$ under vacuum for 1 hour to degas the solvents. Next, the flask containing the $\mathrm{PbBr}_{2}$ was filled with nitrogen gas and brought to a temperature between $80^{\circ} \mathrm{C}$ and $160^{\circ} \mathrm{C}$ depending on the desired NC size. ${ }^{1}$ The Cs-precursor flask was also filled with nitrogen and allowed to cool to $100^{\circ} \mathrm{C}$. Then $0.5 \mathrm{~mL}$ of the Cs-oleate solution was swiftly injected into the $\mathrm{PbBr}_{2}$ containing solution. This reaction mixture was then cooled down to room temperature by immersing in a room temperature water bath. Since NC size is determined by the $\mathrm{Br}: \mathrm{Pb}$ ratio rather than by the kinetics of the reaction, it was not necessary to cool the solution rapidly after adding the Cs-oleate. ${ }^{5}$ 
In order to increase the monodispersity of $\mathrm{NCs}, 10 \mathrm{~mL}$ of anhydrous hexanes (SigmaAldrich, mixture of isomers anhydrous $\geq 99 \%$ ) were added immediately after the reaction mixture had cooled to room temperature. ${ }^{6}$ This disperses all the $\mathrm{CsPbBr}_{3} \mathrm{NCs}$, but does not disperse unreacted precursors or large $\mathrm{Cs} \mathrm{PbBr}_{3}$ aggregates. This turbid mixture was centrifuged and the supernatant containing the synthesized colloidal NCs was saved. Next, dry nitrogen was blown over the surface of the colloid to slowly evaporate off hexanes. Upon sufficient evaporation of hexanes, the largest NCs began to precipitate out of the colloidal solution, which was characterized by a visual change in the solution from transparent to turbid. This occurs as hexanes are a better solvent for $\mathrm{CsPbBr}_{3} \mathrm{NCs}$ than $\mathrm{ODE}$, in which the synthesis is performed. This turbid solution was then centrifuged, with the first NC size fraction in the pellet and the rest of the colloidal NCs remaining in the hexanes/ODE solution. This procedure was repeated until there were no hexanes remaining. NCs from each fraction were resuspended in anhydrous hexanes for storage.

\section{S1.2 Photoluminescence spectroscopy}

The perovskite NCs were resuspended in paraffin oil (Fluka Analytical) to make dilute colloidal solutions. This solvent was used to collect ambient PL spectra of the NCs in the same medium as the high-pressure experiments. Solutions were excited using a continuous wave 406 $\mathrm{nm}$ diode laser focused into the cuvette with an intensity of $0.1 \mathrm{~W} / \mathrm{cm}^{2}$. The resulting PL was collected in a $180^{\circ}$ geometry with a $450 \mathrm{~nm}$ long pass filter and an achromatic doublet pair which focused the PL light onto a multimode optical fiber (Avantes, $400 \mu \mathrm{m}$ core). The spectra were measured using a fiber optic spectrometer (Avantes, 300 lines $/ \mathrm{mm}, 2.5 \mathrm{~nm}$ resolution). The resulting PL spectra are displayed in Figure S1. 


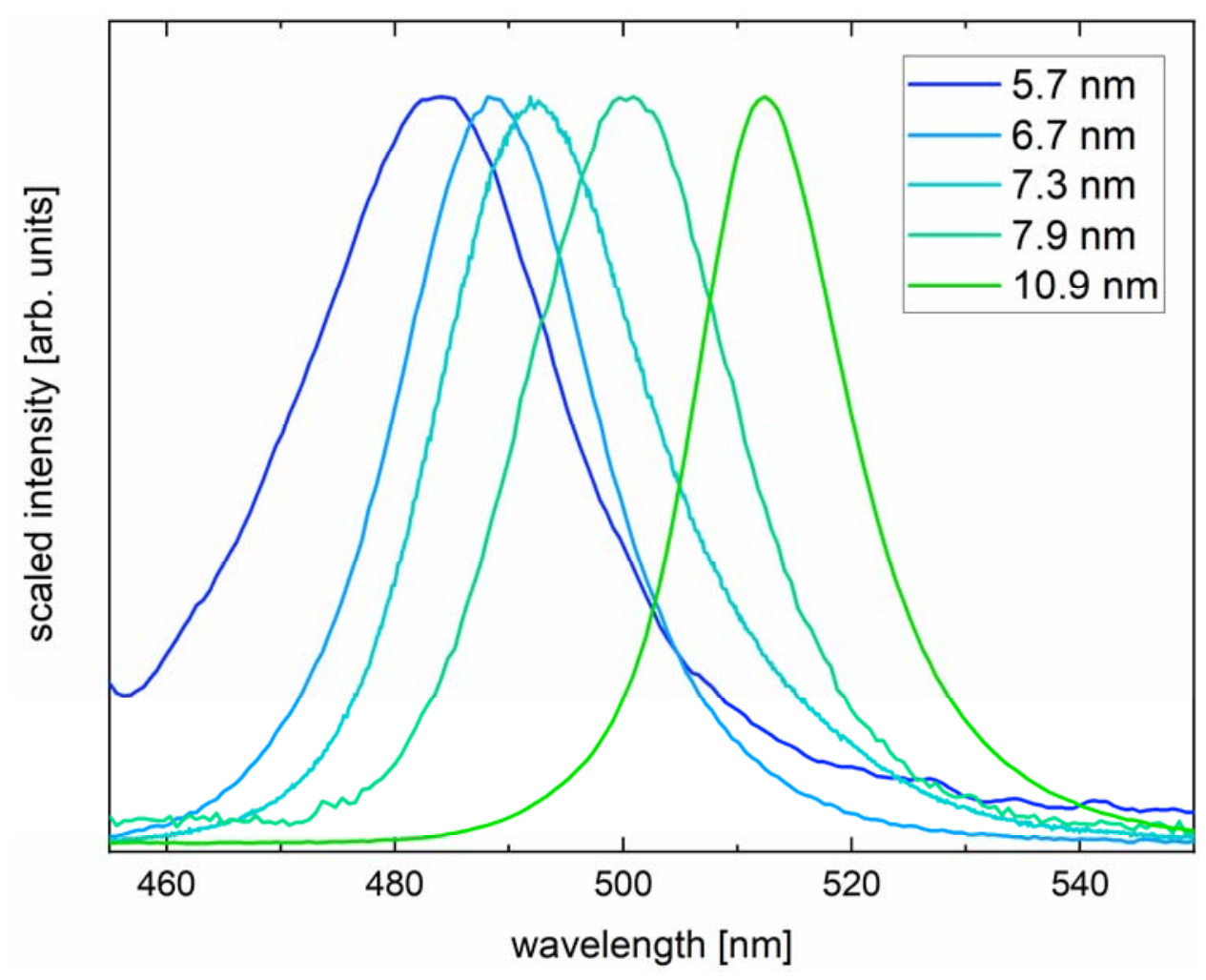

Figure S1: PL spectra of NCs in paraffin oil at ambient pressure.

\section{S1.3 Transmission electron microscopy}

Transmission electron microscopy (TEM) images were acquired using a FEI Tecnai ST20 and a FEI Tecnai F20 with an accelerating voltage of $200 \mathrm{kV}$. TEM samples were prepared by drop casting perovskite $\mathrm{NC}$ in a hexanes solutions onto 300 mesh carbon film copper grids (Electron Microscopy Sciences). The size analyses for all size samples are shown in Figure S2. 


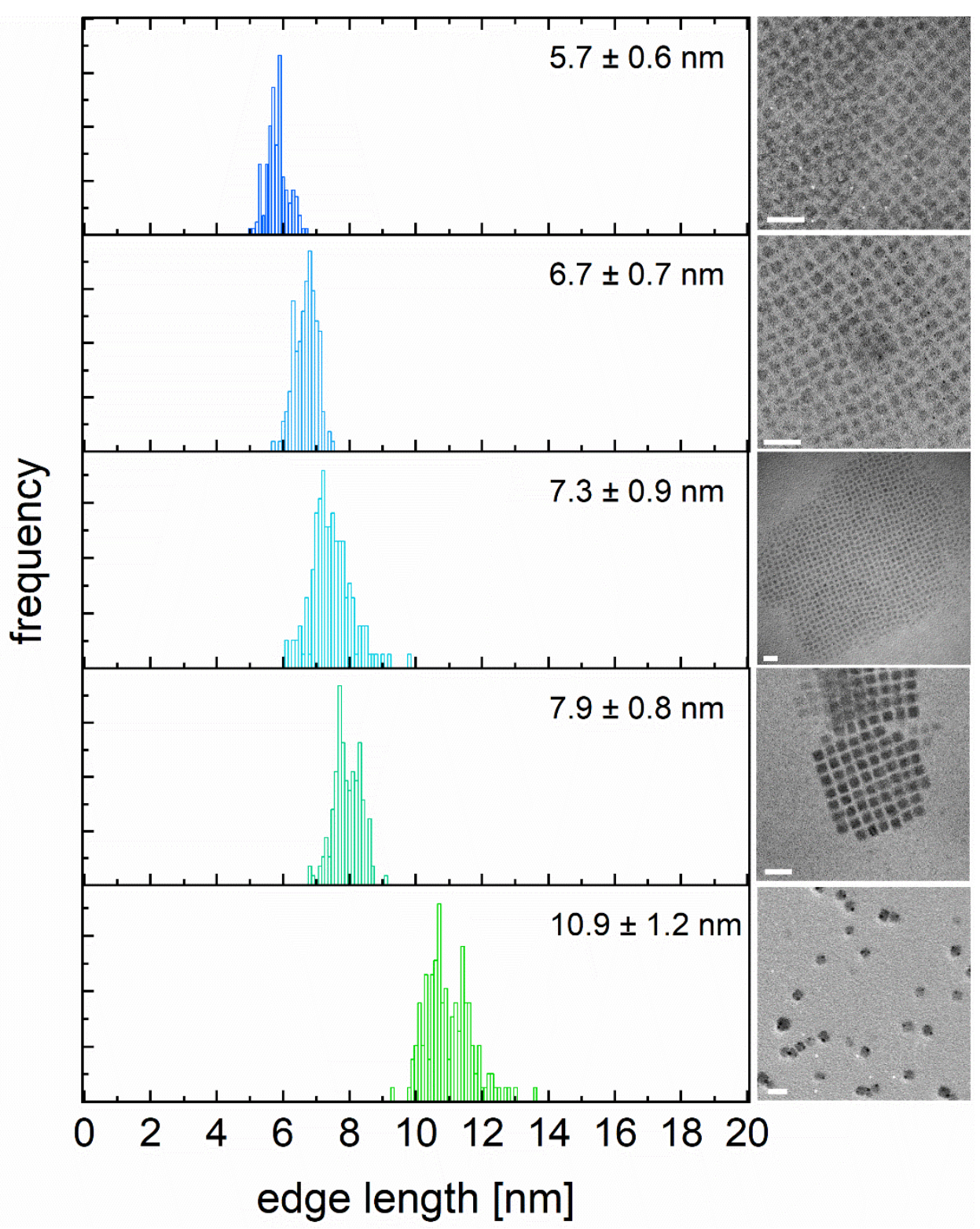

Figure S2: Size distributions and characteristic sample TEM images of all the NC samples studied. Each size distribution was obtained by measuring 150 NCs. All size distributions have $\sim 10 \%$ error which is standard for metal halide perovskite NC samples with the current size selective techniques. $^{8}$ 


\section{S1.4 Powder x-ray diffraction}

Samples for powder $\mathrm{x}$-ray diffraction measurements were prepared by drop casting NC solutions in hexanes onto a Si low background holder (Bruker Si low background sample holder). Diffraction patterns were measured with a Bruker D8 Advance using $\mathrm{Cu} \mathrm{K} \alpha$ radiation with $4^{\circ}$ soller slit pair and a $0.6^{\circ}$ divergence slit.

\section{S1.5 High pressure experiments}

For the high-pressure experiments, aliquots from each size fraction were resuspended in paraffin oil (Fluka Analytical) as a high-pressure transmitting fluid. Paraffin oil remains hydrostatic below $3 \mathrm{GPa}^{9}$ and is a nonpolar solvent that does not degrade the stability of the perovskite NCs. However, similar to ODE, paraffin oil is a poor solvent for perovskite NCs, so very dilute solutions were made that were optically clear to avoid pressurizing aggregates of NCs, which has been shown to permanently sinter them. ${ }^{10}$

High pressure experiments were performed using a gas membrane driven diamond anvil cell (easyLab Diacell $\mu$ ScopeDAC-RT(G) type Ia 16-sided diamond anvils, base diameter $2.5 \mathrm{~mm}$, culet $0.50 \mathrm{~mm}, \mathrm{NA}=0.54)$. High pressure gaskets were made by pre-indenting $250 \mu \mathrm{m}$ thick type 302 stainless steel to a final thickness of $\sim 100 \mu \mathrm{m}$ between the diamonds. A $120 \mu \mathrm{m}$ diameter hole was then laser drilled in the pre-indented gasket as a sample compartment. For each experiment, a small grain of ruby was inserted into the hole, and approximately $2 \mu \mathrm{L}$ droplets of the perovskites in paraffin oil solution were pipetted onto the hole. The position of the $\mathrm{R}_{1}$ fluorescence line of ruby has been calibrated under high pressure such that it can be used as a pressure gauge for diamond anvil cell experiments. ${ }^{11}$ Ruby fluorescence spectra were recorded on a fiber optic spectrometer (Avantes, 1800 lines $/ \mathrm{mm}, 0.1 \mathrm{~nm}$ resolution) and fitted with two Lorentzian peaks to extract the peak position of the $\mathrm{R}_{1}$ and $\mathrm{R}_{2}$ lines for pressure measurements (Figure $\mathrm{S} 3$ ). The error in the fits for the peak position were less than $0.01 \mathrm{~nm}$ for each measurement, which corresponds to an error 
in the pressure measurement ca. $25 \mathrm{MPa}$. The main contribution to the error bars on pressure ( \pm $75 \mathrm{MPa}$ ) is that different ruby grains have different internal strain at ambient pressure, affecting the accuracy of the pressure measurement through the emission wavelengths at ambient conditions.

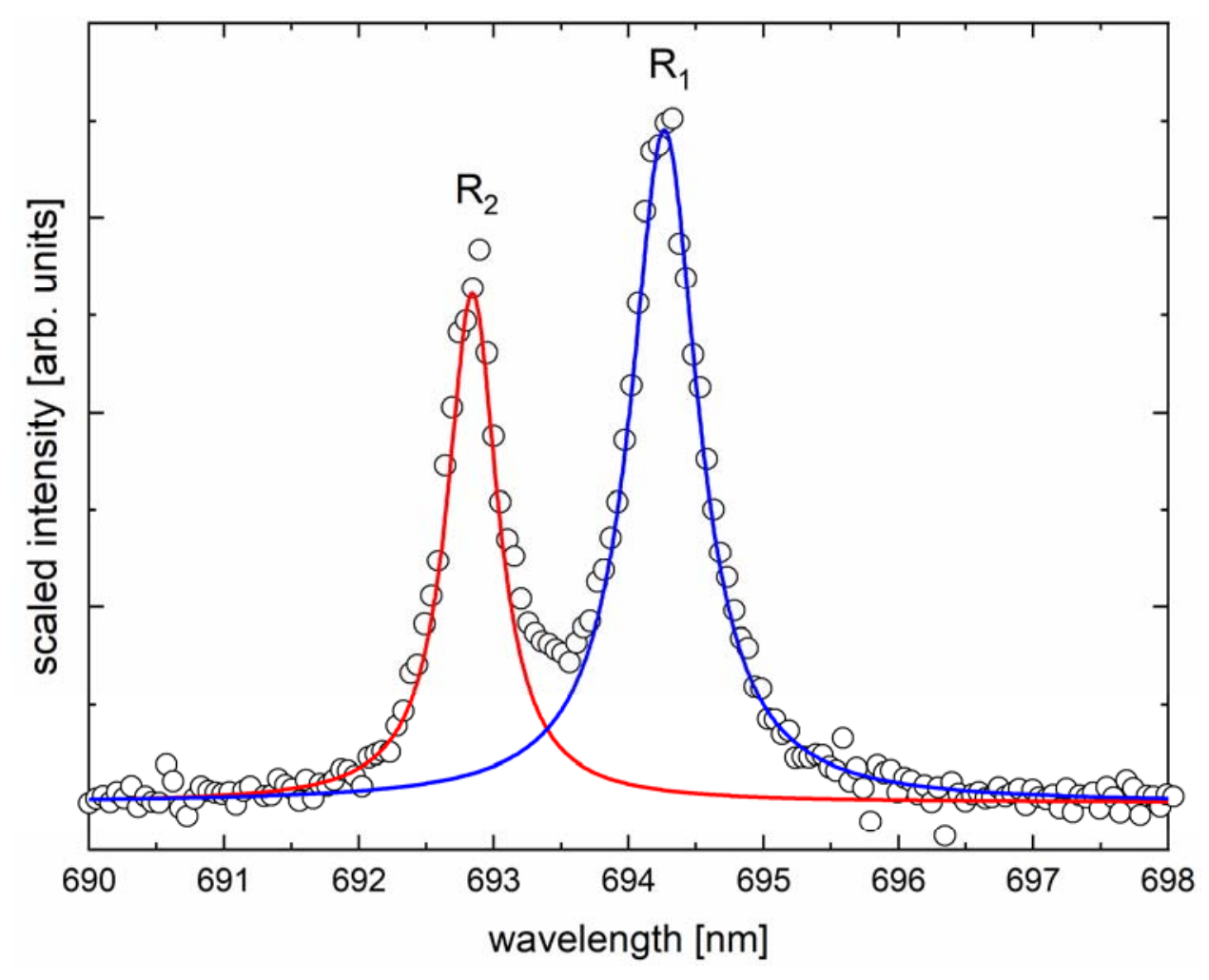

Figure S3: Ruby spectrum with Lorentzian fits of the $\mathrm{R}_{1}$ and $\mathrm{R}_{2}$ peaks for pressure determination.

The perovskite NCs and the ruby grain were excited using a continuous wave $406 \mathrm{~nm}$ diode laser focused into the diamond anvil cell with an intensity of $0.1 \mathrm{~W} / \mathrm{cm}^{2}$. The resulting PL was collected in the same setup described above. At least two high-pressure runs were performed for each NC size. Figures S4-S8 show sample PL spectra taken at elevated pressures, while the data for all high-pressure runs are reported together in Figure 2 of the main text. 


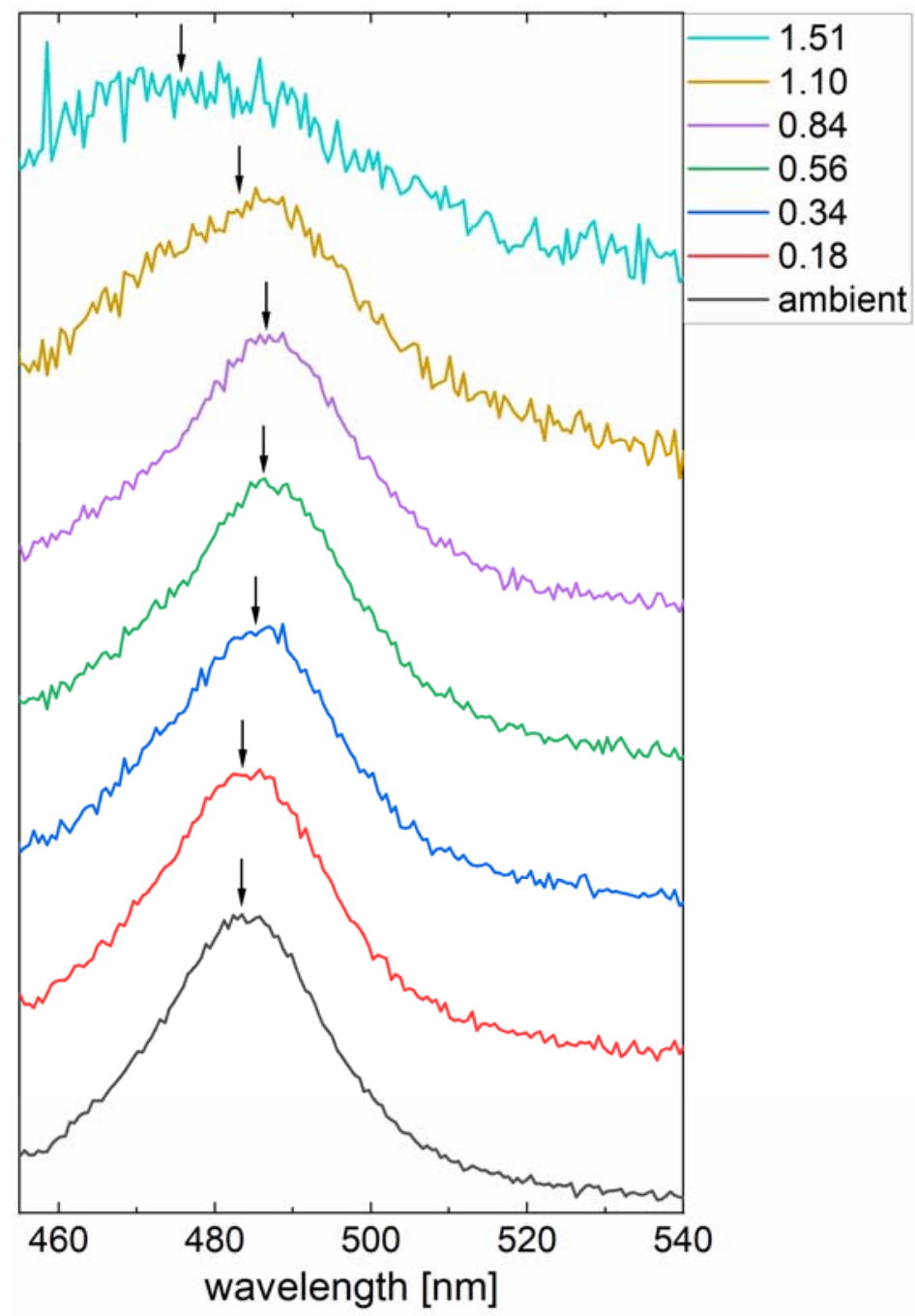

Figure S4: Sample PL spectra for the $5.7 \mathrm{~nm}$ NC sample. Pressures are reported in GPa. Arrows mark the peak center for a single Lorentzian peak fit. 


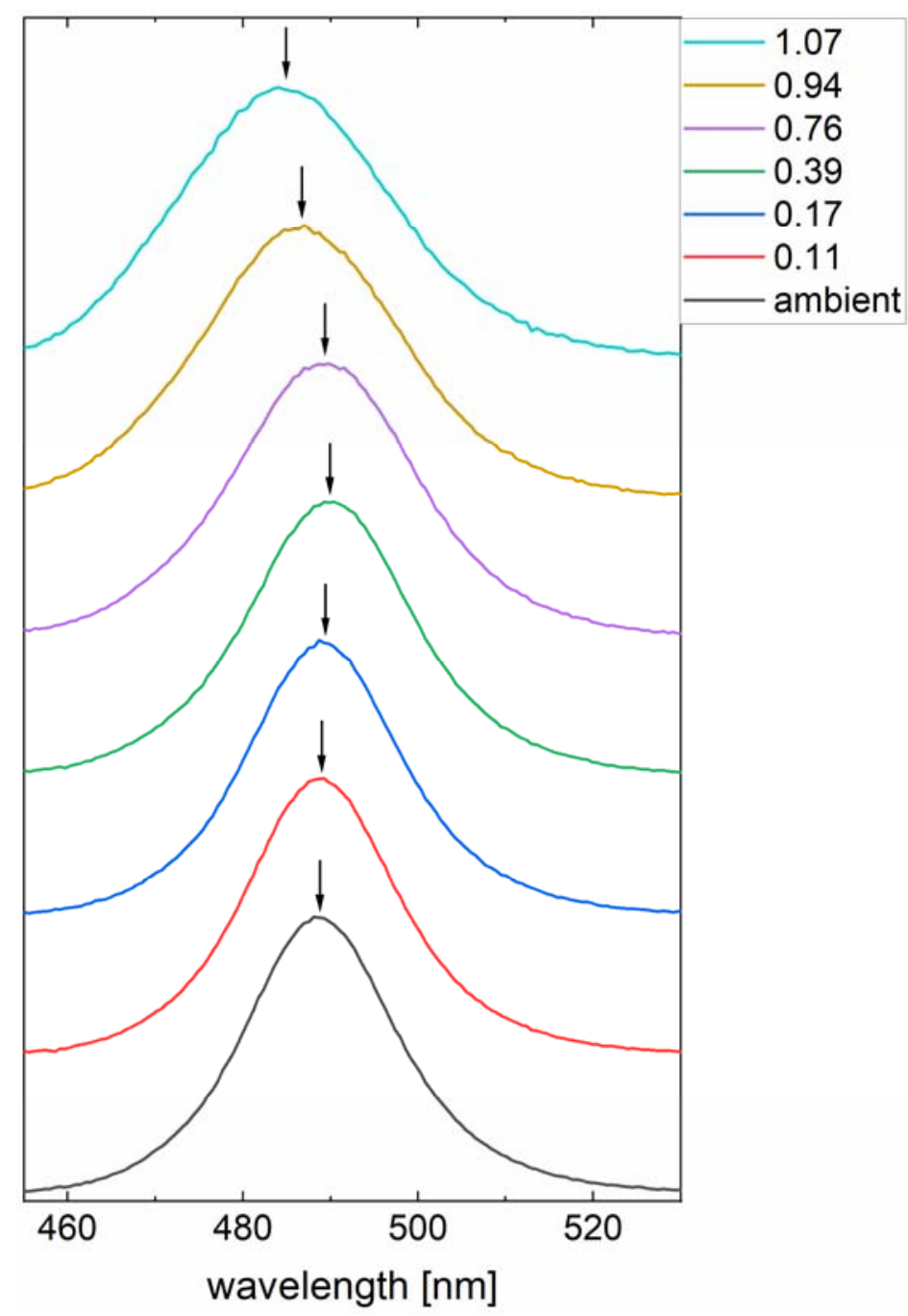

Figure S5: Sample PL spectra for the $6.7 \mathrm{~nm}$ NC sample. Pressures are reported in GPa. Arrows mark the peak center for a single Lorentzian peak fit. 


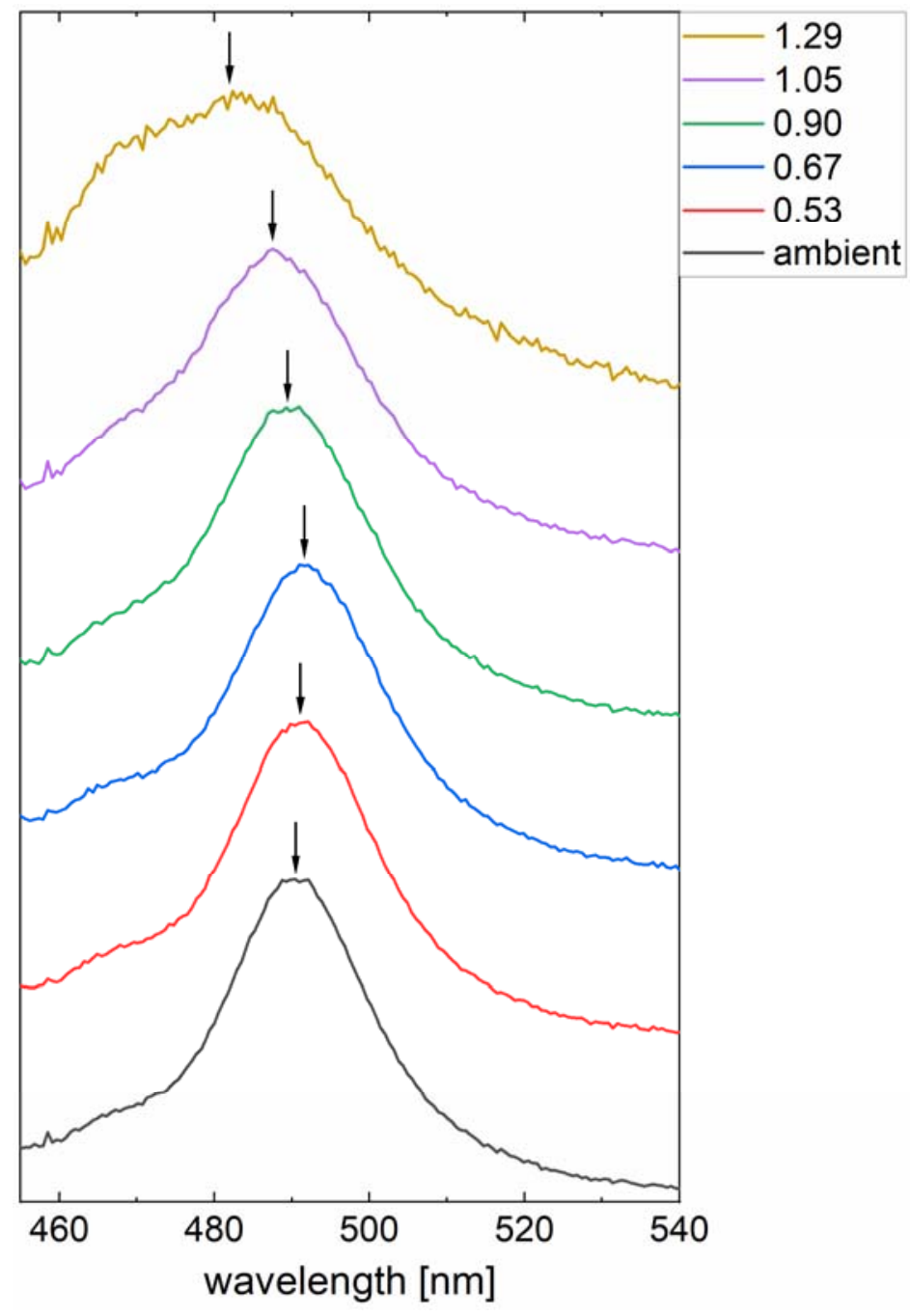

Figure S6: Sample PL spectra for the $7.3 \mathrm{~nm}$ NC sample. Pressures are reported in GPa. Arrows mark the peak center for a single Lorentzian peak fit. 


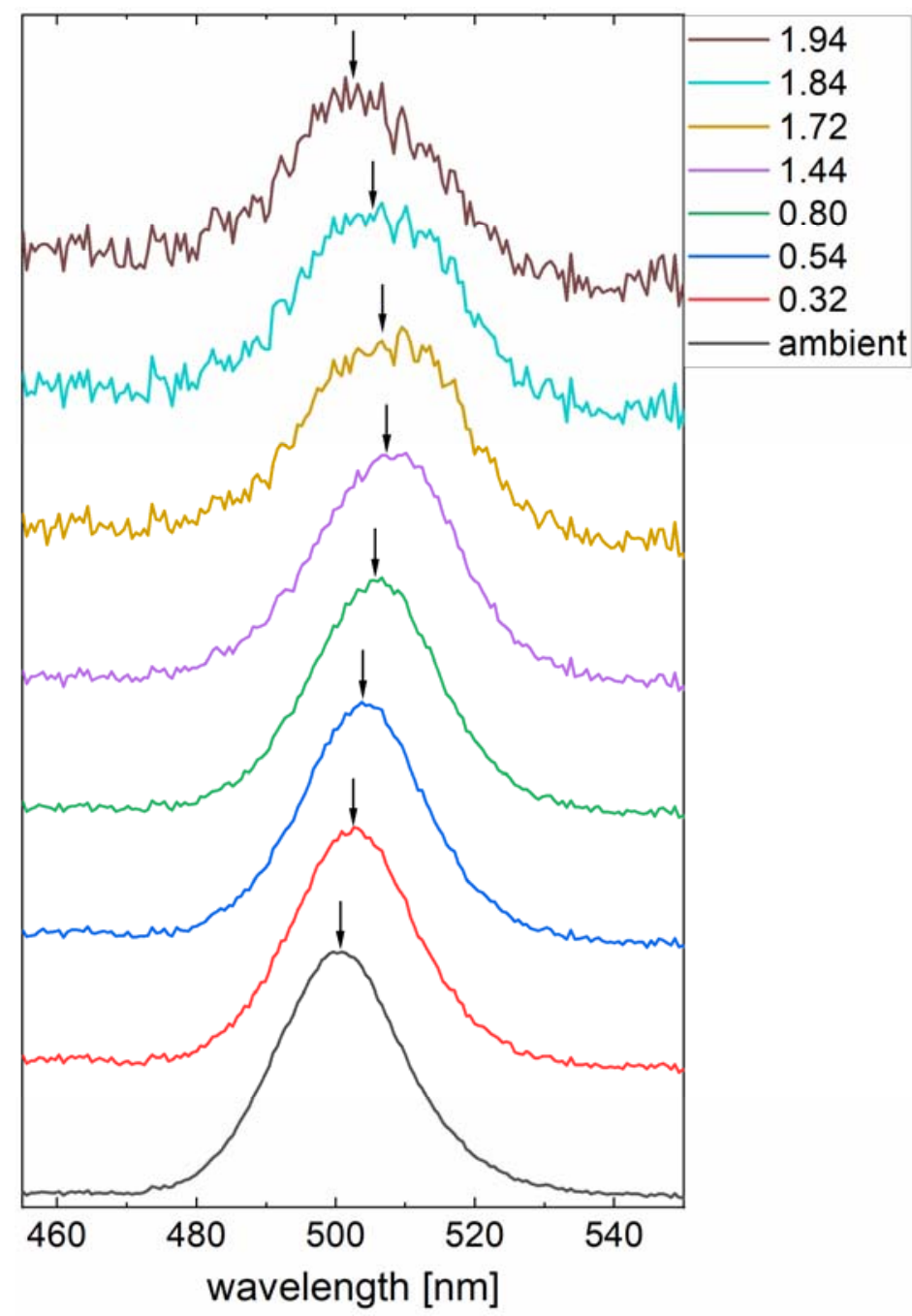

Figure S7: Sample PL spectra for the $7.9 \mathrm{~nm}$ NC sample. Pressures are reported in GPa. Arrows mark the peak center for a single Lorentzian peak fit. 


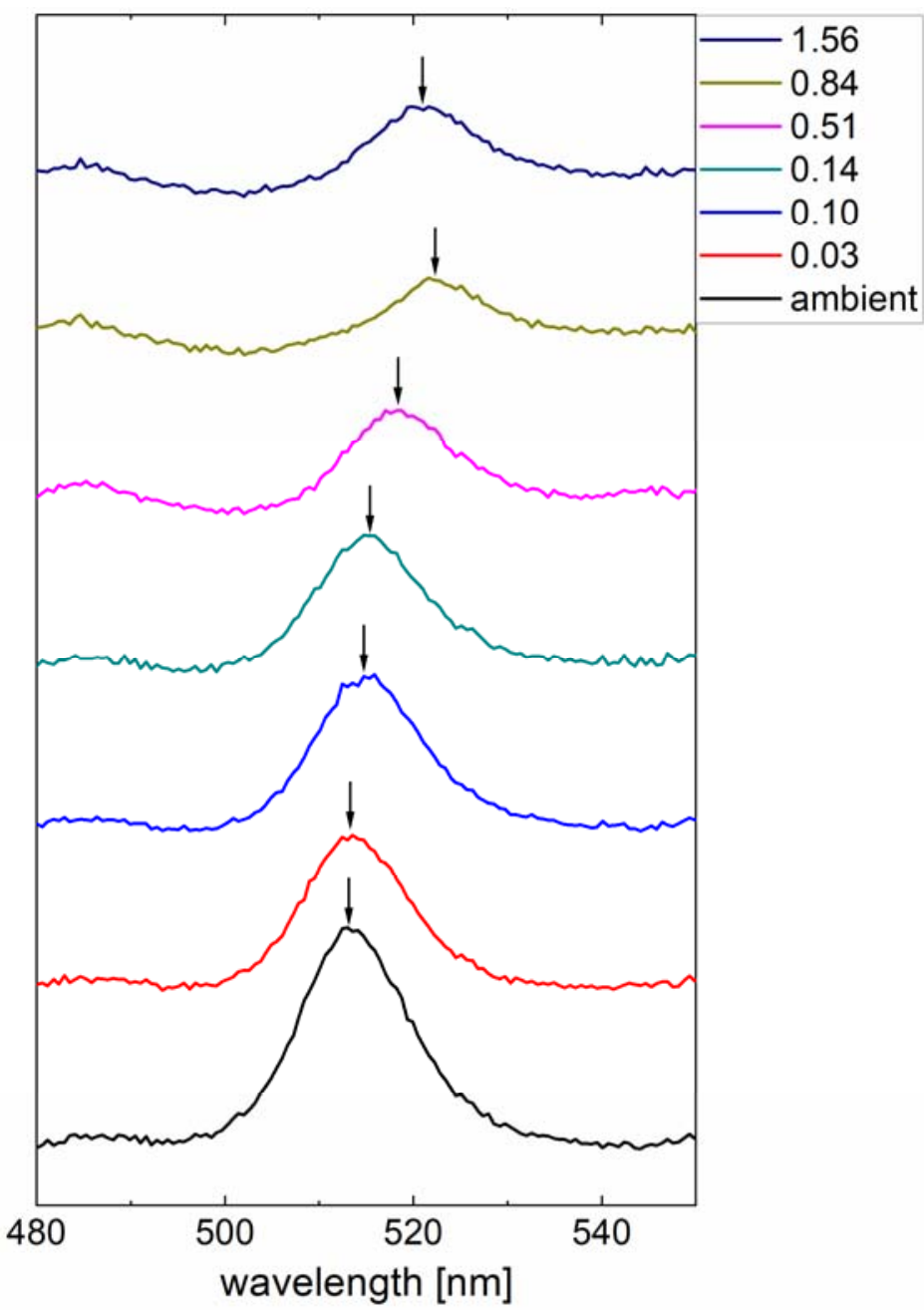

Figure S8: Sample PL spectra for the $10.9 \mathrm{~nm}$ NC sample. Pressures are reported in GPa. Arrows mark the peak center for a single Lorentzian peak fit. The peak centered ca. $485 \mathrm{~nm}$ is fluorescence from the diamonds in the DAC. The diamonds were replaced with synthetic diamonds with low fluorescence background for measurements on all the smaller sizes. 


\section{S2. Supporting Information References}

(1) Dong, Y.; Qiao, T.; Kim, D.; Parobek, D.; Rossi, D.; Son, D. H. Precise Control of Quantum Confinement in Cesium Lead Halide Perovskite Quantum Dots via Thermodynamic Equilibrium. Nano Lett. 2018, 18, 3716-3722.

(2) Protesescu, L.; Yakunin, S.; Bodnarchuk, M. I.; Krieg, F.; Caputo, R.; Hendon, C. H.; Yang, R. X.; Walsh, A.; Kovalenko, M. V. Nanocrystals of Cesium Lead Halide Perovskites $\left(\mathrm{CsPbX}_{3}, \mathrm{X}=\mathrm{Cl}, \mathrm{Br}\right.$, and I): Novel Optoelectronic Materials Showing Bright Emission with Wide Color Gamut. Nano Lett. 2015, 15, 3692-3696.

(3) Dutta, A.; Dutta, S. K.; Das Adhikari, S.; Pradhan, N. Tuning the Size of CsPbBr3 Nanocrystals: All at One Constant Temperature. ACS Energy Lett. 2018, 3, 329-334.

(4) Baranov, D.; Lynch, M. J.; Curtis, A. C.; Carollo, A. R.; Douglass, C. R.; Mateo-Tejada, A. M.; Jonas, D. M. Purification of Oleylamine for Materials Synthesis and Spectroscopic Diagnostics for Trans Isomers. Chem. Mater. 2019, 31, 1223-1230.

(5) Shi, H.; Du, M.-H. Shallow Halogen Vacancies in Halide Optoelectronic Materials. Phys. Rev. B 2014, 90, 174103.

(6) Nenon, D. P.; Pressler, K.; Kang, J.; Koscher, B. A.; Olshansky, J. H.; Osowiecki, W. T.; Koc, M. A.; Wang, L.-W.; Alivisatos, A. P. Design Principles for Trap-Free $\mathrm{CsPbX}_{3}$ Nanocrystals: Enumerating and Eliminating Surface Halide Vacancies with Softer Lewis Bases. J. Am. Chem. Soc. 2018, 140, 17760-17772.

(7) Koscher, B. A.; Swabeck, J. K.; Bronstein, N. D.; Alivisatos, A. P. Essentially Trap-Free $\mathrm{CsPbBr}_{3}$ Colloidal Nanocrystals by Postsynthetic Thiocyanate Surface Treatment. J. Am. Chem. Soc. 2017, 139, 6566-6569.

(8) Zhao, Q.; Hazarika, A.; Schelhas, L. T.; Liu, J.; Gaulding, E. A.; Li, G.; Zhang, M.; Toney, M. F.; Sercel, P. C.; Luther, J. M. Size-Dependent Lattice Structure and Confinement Properties in $\mathrm{CsPbI}_{3}$ Perovskite Nanocrystals: Negative Surface Energy for Stabilization. ACS Energy Lett. 2019, 238-247.

(9) Otto, J. W.; Vassiliou, J. K.; Frommeyer, G. Nonhydrostatic Compression of Elastically Anisotropic Polycrystals. I. Hydrostatic Limits of 4:1 Methanol-Ethanol and Paraffin Oil. Phys. Rev. B 1998, 57, 3253-3263.

(10) Nagaoka, Y.; Hills-Kimball, K.; Tan, R.; Li, R.; Wang, Z.; Chen, O. Nanocube Superlattices of Cesium Lead Bromide Perovskites and Pressure-Induced Phase Transformations at Atomic and Mesoscale Levels. Adv. Mater. 2017, 29, 1606666.

(11) Holzapfel, W. B. Refinement of the Ruby Luminescence Pressure Scale. J. Appl. Phys. 2003, 93, 1813-1818. 